\title{
Non-Self-Similar Dead-Core Rate for the Fast Diffusion Equation with Dependent Coefficient
}

\author{
Liping Zhu' ${ }^{1}$ and Zhengce Zhang ${ }^{2}$ \\ ${ }^{1}$ College of Science, Xi'an University of Architecture \& Technology, Xi'an 710054, China \\ ${ }^{2}$ School of Mathematics and Statistics, Xian Jiaotong University, Xian 710049, China \\ Correspondence should be addressed to Liping Zhu; nyzhuliping@gmail.com
}

Received 24 February 2014; Accepted 26 May 2014; Published 9 June 2014

Academic Editor: Jaan Janno

Copyright (c) 2014 L. Zhu and Z. Zhang. This is an open access article distributed under the Creative Commons Attribution License, which permits unrestricted use, distribution, and reproduction in any medium, provided the original work is properly cited.

We consider the dead-core problem for the fast diffusion equation with spatially dependent coefficient and show that the temporal dead-core rate is non-self-similar. The proof is based on the standard compactness arguments with the uniqueness of the self-similar solutions and the precise estimates on the single-point final dead-core profile.

\section{Introduction}

In this paper, we study the porous medium equation with the following initial-boundary condition:

$$
\begin{gathered}
u_{t}=\left(u^{m}\right)_{x x}-x^{q} u^{p}, \quad(x, t) \in(0,1) \times(0, T), \\
u_{x}(0, t)=0, \quad u(1, t)=k, \quad t \in(0, T), \\
u(x, 0)=u_{0}(x), \quad x \in[0,1],
\end{gathered}
$$

where $0<p<m<1$ and $-1<q<0$. Assume $k>0$ and that the initial data $u_{0}$ satisfies

$$
u_{0}>0 \text { in }[0,1], \quad u_{0}^{\prime}(0)=0, \quad u_{0}(1)=k .
$$

We set

$$
\theta(t):=\min _{0 \leq x \leq 1} u(x, t)
$$

and denote

$$
T=T\left(u_{0}\right):=\inf \{t>0 ; \theta(t)=0\}>0 .
$$

Moreover, we denote

$$
\beta=\frac{q+2}{2(1-p)+q(1-m)} .
$$

It is called that $u$ develops a dead-core in a finite time if $T<$ $\infty$, and it is shown that $u$ develops a dead-core in finite time for certain initial-boundary data (see [1, Theorem 1.1]).
Suppose that $u$ develops a dead-core in the finite time $T$. Our main purpose of this paper is to study the temporal deadcore rate as $t \rightarrow T$. For our main results on the asymptotic dead-core behaviour, we will assume that $u_{0}$ satisfies the following conditions:

$$
\begin{aligned}
& u_{0} \in C^{2}([0,1]), \quad\left(u_{0}^{m}\right)^{\prime \prime} \leq x^{q} u_{0}^{p} \text { in }(0,1], \\
& u_{0} \text { is nondecreasing in } x \text { and } T\left(u_{0}\right)<\infty .
\end{aligned}
$$

It then follows from the strong maximum principle that $u_{t}<$ 0 in $Q_{T}:=(0,1) \times(0, T)$ and $u_{x}>0$ in $Q_{T}$. Here, we introduce the following self-similar transformation:

$$
\begin{gathered}
y=\frac{x}{(T-t)^{\alpha}}, \quad s=-\ln (T-t), \\
v(y, s)=\left[\frac{u(x, t)}{(T-t)^{\beta}}\right]^{m},
\end{gathered}
$$

where $\alpha=(m-p) /[2(1-p)+q(1-m)]$. Then $v$ satisfies

$$
\begin{gathered}
\frac{1}{m} v^{(1 / m)-1} v_{s} \\
=v_{y y}-\frac{\alpha}{m} y v^{(1 / m)-1} v_{y}+\beta v^{1 / m}-y^{q} v^{p / m}, \quad \text { in } \Omega, \\
v_{y}(0, s)=0, \quad v\left(e^{\alpha s}, s\right)=k^{m} e^{\beta m s}, \quad s>s_{0}, \\
v\left(y, s_{0}\right)=v_{0}(y)=T^{-\beta m} u_{0}^{m}\left(y T^{\alpha}\right), \quad y \in\left[0, T^{-\alpha}\right], \\
\text { where } \Omega:=\left\{(y, s) \mid 0<y<e^{\alpha s}, s>s_{0}=-\ln T\right\} .
\end{gathered}
$$


The dead-core problem for the homogeneous equation

$$
u_{t}=\Delta u^{m}-u^{p}
$$

in the general higher spatial dimensional case has been studied extensively in the past years. We refer the reader to, for example, [2-9] and the references therein. In particular, for the slow diffusion (i.e., $0<p<1<m$ ) and $m+p \geq 2$ case, the self-similar singularity of dead-core rate $(T-t)^{1 /(1-p)}$ was shown in [5] for certain class of initial data. On the other hand, for the fast diffusion case (i.e., $0<p<m<1$ ) or semilinear case (i.e., $m=1$ ), it is shown that the temporal dead-core rate is non-self-similar in the sense that it is always faster than the self-similar rate. Moreover, the exact dead-core rates (depending on the initial data) have been derived for the semilinear heat equation in [10] for radial symmetric case. Other singularity formation mechanisms in related reactiondiffusion equations and reaction-convection equations, such as type II blowup and gradient blowup, also exhibit non-selfsimilar behaviours, respectively. We refer to $[11,12]$ for type II blowup and to [13-21] for gradient blowup for recent related results.

To study the dead-core rate for (1) with a spatially dependent absorption term, we assume further that $q \in$ $(-1,0)$ and $q \geq-2 p / m$.

Let

$$
\begin{gathered}
V_{0}(y):=C_{0} y^{m(q+2) /(m-p)}, \quad y>0, \\
C_{0}:=C_{0}(p, m, q)=\left[\frac{(m-p)^{2}}{m(m+p+m q)(q+2)}\right]^{m /(m-p)}>0 .
\end{gathered}
$$

By a limiting process with some a priori estimates, we prove the following main theorem of this paper.

Theorem 1. Assume that $0<p<m<1, p+m>1$, and $-1<q<0$ such that $q \geq-2 p / m$ and that (6) holds. Then we have $\lim _{s \rightarrow \infty} v(y, s)=V_{0}(y)$ uniformly for $y \in[0, M]$ for any $M>0$. Moreover,

$$
\lim _{t \rightarrow T^{-}}(T-t)^{-\beta} \theta(t)=0 .
$$

Note that the function $V_{0}$ is a stationary solution of the equation in $(8)$ in $(0, \infty)$ with $V(0)=0$. As a consequence, Theorem 1 implies that the dead-core rate is non-self-similar. Also, there is no constant stationary solution of (8) due to the spatially dependent nonlinearity. Indeed, we prove in Section 2 that the only nontrivial nondecreasing nonnegative stationary solution of $(8)$ in $(0, \infty)$ with polynomial bound is $V_{0}$.

One of the reasons to consider the dead-core problem with variable coefficient is to investigate the effect of degeneracy on the dead-core phenomenon. In fact, a related blow-up problem for the singular equation

$$
x^{q} u_{t}=u_{x x}+u^{p}, \quad 0<x<1, t>0,
$$

with $q>0, p>1$ and the Dirichlet boundary condition, has been studied by Floater [22] and Lacey [23]. Equation (12) arises from Ockendon's model for the flow in a channel of a fluid whose viscosity is temperature dependent (see $[22,23]$ ). More generally, Wang and Zheng [24] investigated the critical Fujita exponent, that is, $p_{c}=m+\left(2+\lambda_{2}\right) /\left(N+\lambda_{1}\right)$, for the initial-value problem of the degenerate and singular nonlinear parabolic equation:

$$
|x|^{\lambda_{1}} u_{t}=\Delta u^{m}+|x|^{\lambda_{2}} u^{p}, \quad x \in \mathbb{R}^{N}, t>0,
$$

with a nonnegative initial value, where $p>m \geq 1$ and $0 \leq$ $\lambda_{1} \leq \lambda_{2}<p\left(\lambda_{1}+1\right)-1$.

There are certain difficulties in dealing with the spatially dependent absorption term. For example, (1) is not translation invariant due to the spatially dependent nonlinearity. In fact, Wu and Zhang [25] studied the semilinear case of (1) with $m=1$ and proved that the temporal dead-core rate is non-self-similar, but they did not get the uniqueness of dead-core points. In the present paper, based on Lemma 3.1 in [1] which is derived by using an integral form of maximum principles, we will obtain a precise estimate on dead-core profile and prove that the dead-core is a single point and show that the exponent $q$ essentially affects the asymptotic behaviours of dead-core. Therefore, the proofs in this paper are more delicate than those in $[6,25]$.

This paper is organized as follows. The uniqueness of stationary solution of (8) is proved in Section 2. In Section 3, we derive some a priori estimates. Finally, we prove the main theorem (Theorem 1) in Section 4.

\section{Uniqueness of Self-Similar Solutions}

In this section, we will prove that the only nontrivial nondecreasing nonnegative stationary solution of $(8)$ in $(0, \infty)$ with polynomial bound is $V_{0}$.

Lemma 2. Let $V \in C^{2}(0, \infty)$ be a solution of the stationary equation of (8):

$$
V_{y y}-\frac{\alpha}{m} y V^{(1 / m)-1} V_{y}+\beta V^{1 / m}-y^{q} V^{p / m}=0, \quad y>0,
$$

such that $V>0, V^{\prime} \geq 0$, and $V$ is polynomially bounded. Then $V=V_{0}$.

Proof. Set

$$
W(y)=V^{\tau}(y) \quad \text { with } \tau=\frac{2(m-p)}{m(q+2)}, y>0,
$$

for a given positive solution $V$ of (14). Then, at any point $y>0$ with $W(y)>0, W$ satisfies the equation

$$
\begin{aligned}
W^{\prime \prime} & -\frac{\alpha}{m} y W^{(1-m) / m \tau} W^{\prime}+\tau \beta W^{((1-m) / m \tau)+1}+\frac{1-\tau}{\tau} \frac{\left(W^{\prime}\right)^{2}}{W} \\
& =\tau y^{q} W^{-q / 2}, \quad y>0 .
\end{aligned}
$$


Differentiating (16) once, we obtain

$$
\begin{aligned}
W^{\prime \prime \prime} & -\frac{\alpha}{m} y W^{(1-m) / m \tau} W^{\prime \prime}+\left[\left(\frac{1-m}{m}+\tau\right) \beta-\frac{\alpha}{m}\right] \\
& \times W^{(1-m) / m \tau} W^{\prime}-\frac{(1-m) \alpha}{m^{2} \tau} y W^{(1-(\tau+1) m) / m \tau}\left(W^{\prime}\right)^{2} \\
= & q \tau y^{q-1} W^{-(q / 2)-1}\left(W-\frac{y W^{\prime}}{2}\right) \\
& -\frac{1-\tau}{\tau} \frac{W^{\prime}\left[2 W W^{\prime \prime}-\left(W^{\prime}\right)^{2}\right]}{W^{2}}
\end{aligned}
$$

for $y>0$. As in [6], we introduce the functions

$$
H:=W-\frac{y}{2} W^{\prime}, \quad Z:=|H|^{1 / \tau} .
$$

After a calculation similar to [6], by using (17) we obtain, for $y \in D$,

$$
D:=\{y>0 W(y)>0, H(y) \neq 0\} ;
$$

the function $Z$ satisfies

$$
\begin{aligned}
Z^{\prime \prime}-\frac{\alpha}{m} y W^{(1-m) / m \tau} Z^{\prime} & =\frac{1}{\tau}|H|^{(1 / \tau)-2} \\
\times & \left\{-\frac{q \tau}{2} y^{q} W^{-(q / 2)-1}\left(W-\frac{y}{2} W^{\prime}\right)^{2}\right. \\
& +\frac{2 p+m q}{8(m-p)}\left[W^{\prime}+y\left(\frac{W W^{\prime \prime}-\left(W^{\prime}\right)^{2}}{W}\right)\right]^{2} \\
& \left.+\frac{(1-m) \beta}{2 m} y W^{(1-(\tau+1) m) / m \tau}\left(W-\frac{y W^{\prime}}{2}\right)^{2} W^{\prime}\right\} .
\end{aligned}
$$

Assume that $2 p+m q \geq 0$; that is, for the given $p, m \in(0,1)$ such that $0<p<m<1$, we require

$$
q \in\left[\frac{-2 p}{m}, 0\right) \subset(-2,0) \text {. }
$$

Then we deduce from (20) that $\left(e^{-\rho(y)} Z^{\prime}\right)^{\prime} \geq 0$ in $D$, where $\rho(y):=\int_{0}^{y}(\alpha / m) s W^{(1-m) / m \tau}(s) d s$. From this, using the polynomial bound of $V$ (and so is $Z$ ) we can argue as the arguments used in [6] to prove that $D=\{y>0 \mid H(y) \neq 0\}$ and $Z$ is a constant function defined in $(0, \infty)$. Since the proof is very similar to that in [6], we safely omit the details here. Hence $H$ is constant in $(0, \infty)$. By an integration, we end up with that $W(y)=A+B y^{2}$ for $y \in(0, \infty)$ for some constants $A$ and $B$.

Next, we plug the expression $V(y)=\left(A+B y^{2}\right)^{1 / \tau}$ into (14). Then we can easily derive that

$$
A=0, \quad B=\left[\frac{(m-p)^{2}}{m(m+p+m q)(q+2)}\right]^{2 /(q+2)} .
$$

This proves that $V_{0}$ is the only positive solution of (8) in $(0, \infty)$ such that it grows at most polynomially.

\section{A Priori Estimates}

We will derive some a priori estimates in this section. Suppose that the solution $u$ of (1)-(2) with (6) develops a dead-core in the finite time $T$. We have the following precise estimates on the single-point final dead-core profile near $x=0$.

Lemma 3. Assume that $0<p<m<1, p+m>1,-1<q<$ 0 , and the assumptions of (2) and (6) hold. Then there exist $c, C>0$ such that

$$
c x^{(q+2) /(m-p)} \leq u(x, T) \leq C x^{(q+2) /(m-p)}, \quad 0 \leq x \leq 1 .
$$

Proof. By Lemma 3.1 in [1], for any fixed $t_{0} \in(0, T)$, there exists $0<\varepsilon \leq(p+m-1) /[(2 p+m-1)(q+1)]$ such that the auxiliary function

$$
J:=\left(u^{m}\right)_{x}-\varepsilon x^{q+1} u^{p}
$$

satisfies $J \geq 0$ in $[0,1] \times\left(t_{0}, T\right)$. Integrating $J \geq 0$ on $[0, x]$, we get $u(x, t) \geq c x^{(q+2) /(m-p)},(x, t) \in(0,1) \times\left(t_{0}, T\right)$. The lower estimate immediately follows by letting $t \rightarrow T$, where $c:=$ $c(\varepsilon)=\{\varepsilon(m-p) /[m(q+2)]\}^{1 /(m-p)}$. Furthermore, using selfsimilar variables, we have, for the fixed $t_{0} \in(0, T)$,

$$
\begin{gathered}
v(y, s) \geq c^{*} y^{m(q+2) /(m-p)} \quad \text { for } 0<y<e^{\alpha s}, \\
-\ln \left(T-t_{0}\right)<s<\infty,
\end{gathered}
$$

where $c^{*}>0$. On the other hand, since $u_{t}<0$ and $u_{x}>0$ in $Q_{T}$, we have $\left(u^{m}\right)_{x x}<x^{q} u^{p}$. Let $z=u^{m}$. Then from $z_{x}=$ $m u^{m-1} u_{x}, 0<u \leq k$ in $Q_{T}$ and

$$
z_{x x}(x, t)<x^{q} z^{r}(x, t) \quad \text { with } r=\frac{p}{m},(x, t) \in Q_{T} .
$$

Integrating the inequality $z_{x x}(x, t)<x^{q} z^{r}(x, t)$ using $z_{x} \geq 0$, we obtain

$$
\begin{aligned}
z_{x}(x, t) & =\int_{0}^{x} z_{x x}(y, t) d y \leq \int_{0}^{x} y^{q} z^{r}(y, t) d y \\
& \leq z^{r}(x, t) \int_{0}^{x} y^{q} d y \leq \frac{x^{q+1}}{q+1} z^{r}(x, t) .
\end{aligned}
$$

Hence $z^{1-r}(x, t)-z^{1-r}(0, t) \leq((1-r) /(q+1)(q+2)) x^{q+2}$. Consequently,

$$
u(x, t) \leq\left[u(0, t)^{m-p}+\frac{m-p}{m(q+1)(q+2)} x^{q+2}\right]^{1 /(m-p)}
$$

for $(x, t) \in(0,1) \times(0, T]$. In terms of self-similar variables, we deduce from (27) and (28) that

$$
\begin{gathered}
v_{y}(y, s) \leq C_{1} y^{q+1} v^{p / m}(y, s), \\
v(y, s) \leq\left[v^{(m-p) / m}(0, s)+C_{2} y^{q+2}\right]^{m /(m-p)}
\end{gathered}
$$

for $0<y<e^{\alpha s}, s \geq s_{0}$ for some positive constants $C_{1}$ and $\mathrm{C}_{2}$. 
To derive an upper bound for $v(0, s)$, we note that $u_{x x}(0, t)>0$ for all $t \in(0, T]$ by the Hopf boundary point lemma. Then $u_{x x}(0, t) \geq c$ for all $t \in[T / 2, T]$ for some small positive constant $c$. Since $u_{x}$ is uniformly bounded, it follows from the regularity of $u$ that $u_{x x}(x, t)>0$ for $(x, t) \epsilon$ $[0, \delta] \times[T / 2, T]$ for some small positive constant $\delta$. Hence from (1) it follows that

$$
u_{t}(x, t) \geq-x^{q} u^{p}(x, t) \quad \text { for }(x, t) \in(0, \delta] \times\left[\frac{T}{2}, T\right] .
$$

By an integration from $t \in[T / 2, T)$ to $T$, we deduce that

$$
\begin{array}{r}
u(x, t) \leq C_{3} x^{q /(1-p)}(T-t)^{1 /(1-p)} \\
\text { for }(x, t) \in(0, \delta] \times\left[\frac{T}{2}, T\right]
\end{array}
$$

for some positive constant $C_{3}=C_{3}(p)$. Recall that $v$ is increasing in $y$. Taking $x(t)=(T-t)^{\alpha}$ for $0<T-t \ll 1$, we conclude from $(32)$ that $v(0, s) \leq v(1, s) \leq C_{3}$ for $s \gg 1$ and so we obtain the following estimate:

$$
v(0, s) \leq C_{4} \quad \text { for } s \geq s_{0}
$$

for some positive constant $C_{4}$. In particular, (33) and (30) imply that $v$ grows at most polynomially:

$$
\begin{array}{r}
v(y, s) \leq\left[C_{4}^{(m-p) / m}+C_{2} y^{q+2}\right]^{m /(m-p)} \\
\text { for } 0<y<e^{\alpha s}, \quad s \geq s_{0} .
\end{array}
$$

Since $u(0, T)=0$, we also obtain from (28) that

$$
u(x, T) \leq C x^{(q+2) /(m-p)}, \quad x \in[0,1],
$$

where $C:=\{(m-p) /[m(q+1)(q+2)]\}^{1 /(m-p)}$. This gives an upper bound for the dead-core profile.

\section{Proof of Theorem 1}

In this section, we study the asymptotic behaviour of $v(y, s)$ as $s \rightarrow \infty$. For this, we define the energy functional by

$$
E(s):=\int_{0}^{R(s)} \Phi\left(y, v(y, s), v_{y}(y, s)\right) d y,
$$

where $R(s):=e^{\alpha s}$,

$$
\begin{aligned}
\Phi(y, v, w):= & \int_{0}^{w}(w-\sigma) P(y, v, \sigma) d \sigma \\
& -\int_{\mathcal{K}}^{v} \hat{g}(y, \mu) P(y, \mu, 0) d \mu,
\end{aligned}
$$

and $P(y, v, w):=\exp \left\{(-\alpha / m) \int_{0}^{y} \xi \psi(\xi ; y, v, w)^{(1 / m)-1} d \xi\right\}$. Moreover, $\psi$ is defined as the solution of the problem:

$$
\begin{gathered}
\psi_{\xi \xi}-\frac{\alpha}{m} \xi \psi^{(1 / m)-1} \psi_{\xi}+\widehat{g}(\xi, \psi)=0, \quad \xi<y, \\
\psi(y ; y, v, w)=v, \quad \psi_{\xi}(y ; y, v, w)=w,
\end{gathered}
$$

where $v>0, w \in \mathbb{R}$ and $\hat{g}(\xi, v)$ is a smooth cut-off function (defined as in [6]) of $g(y, v):=\beta v^{1 / m}-y^{q} v^{p / m}$ on $\mathbb{R}$. Since $q \in(-1,0)$, the function $y^{q}$ is integrable at $y=0$ and so $E(s)$ is well defined. Following [6], we know that the solution $\psi$ of (38) can be continued backward to $\xi=0$. Moreover, by a simple computation, we obtain that

$$
\begin{aligned}
\frac{d E}{d s}(s)=-\frac{1}{m} \int_{0}^{R(s)} & P\left(y, v(y, s), v_{y}(y, s)\right) v^{(1 / m)-1} \\
& \times v_{s}^{2}(y, s) d y+J(s)
\end{aligned}
$$

where $J$ satisfies the property $\int_{s_{0}}^{\infty}|J(s)| d s<\infty$. From this, we have $(d / d s)\left[E(s)-\int_{s_{0}}^{s} J(\gamma) d \gamma\right] \leq 0$ and therefore for any $s>s_{0}$,

$$
\begin{gathered}
E(s) \leq E\left(s_{0}\right)+\int_{s_{0}}^{s} J(\gamma) d \gamma \leq E\left(s_{0}\right) \\
+\int_{s_{0}}^{\infty} J(\gamma) d \gamma \equiv \widehat{C}<\infty .
\end{gathered}
$$

On the other hand, due to (25), we also obtain from (36) that

$$
\begin{gathered}
\frac{1}{m} \int_{s_{0}}^{\infty} \int_{0}^{R(s)} P\left(y, v(y, s), v_{y}(y, s)\right) v^{(1 / m)-1} \\
\times v_{s}^{2}(y, s) d y d s \leq \widetilde{C}<\infty
\end{gathered}
$$

Note that $P(y, v, w)$ is bounded below away from 0 for $y, v, w$ in bounded sets. Also, by (25), (29), and (34), we can derive for $0<\lambda<1, M>e^{\alpha s_{0}}$ and some $s_{M}$

$$
\int_{s_{M}}^{\infty} \int_{\lambda}^{M} v_{s}^{2}(y, s) d y d s<\infty
$$

Then by a standard limiting process, that is, compactness arguments, we can show that for any sequence $\left\{s_{j}\right\}$ tending to infinity the limit function $v_{\infty}(y, s):=\lim _{j \rightarrow \infty} v\left(y, s+s_{j}\right)$ is such that $v_{\infty}(y, s)=v_{\infty}(y)$ and is a nontrivial nondecreasing nonnegative stationary solution of $(8)$ in $(0, \infty)$. Then the uniqueness of self-similar solutions gives that $v_{\infty}=V_{0}$. Therefore, the conclusion follows from (25) and (34) and Lemma 2. This completes the proof of Theorem 1.

Remark 4. We note that when $q<0$ we here only consider the special case of $-1<q<0$ which can ensure the existence of smooth classical solutions by the parabolic regularity theory. For the case of $q=0$, Guo et al. [6] have studied the existence of the non-self-similar dead-core rate of the solution. However, if $q>0$, from the proof of Theorem 1.1 in [1] (or Theorem 2.1 in [25] for the semilinear equation) we know that the dead-core would not occur at the origin, but it may occur at some point away from the origin if the solution $u$ is not strictly increasing in $x$. Also, it is very interesting whether the non-self-similar dead-core rate exists in this case. We leave this open question to the interested readers. Furthermore, in the higher dimensional space, it is worth to study whether assumptions could make 
the solution $u$ of problem (1) develop a dead-core at the origin or not. Recently, there are some interesting results of blowup problems related to these dead-core questions; we refer to [26] and the references therein for the reaction-diffusion equation with more general variable coefficient.

\section{Conflict of Interests}

The authors declare that there is no conflict of interests regarding the publication of this paper.

\section{Acknowledgments}

The authors thank Professor Jong-shenq Guo for helpful discussions. This work was supported in part by the National Natural Science Foundation of China (no. 11371286), the Scientific Research Foundation for the Returned Overseas Chinese Scholars, State Education Ministry, the Special Fund of Education Department (no. 2013JK0586), and the Youth Natural Science Grant (no. 2013JQ1015) of Shaanxi province of China.

\section{References}

[1] Z. C. Zhang and B. Wang, "Spatial profile of the dead core for the fast diffusion equation with dependent coefficient," International Journal of Differential Equations, vol. 2011, Article ID 751969, 9 pages, 2011.

[2] C. Bandle, T. Nanbu, and I. Stakgold, "Porous medium equation with absorption," SIAM Journal on Mathematical Analysis, vol. 29, no. 5, pp. 1268-1278, 1998.

[3] C. Bandle and I. Stakgold, "The formation of the dead core in parabolic reaction-diffusion problems," Transactions of the American Mathematical Society, vol. 286, no. 1, pp. 275-293, 1984.

[4] L. Wang and Q. Chen, "On the dead core behavior for a semilinear heat equation," Mathematica Applicata, vol. 10, no. 1, pp. 22-25, 1997.

[5] X. Chen, J.-S. Guo, and B. Hu, "Dead-core rates for the porous medium equation with a strong absorption," Discrete and Continuous Dynamical Systems B. A Journal Bridging Mathematics and Sciences, vol. 17, no. 6, pp. 1761-1774, 2012.

[6] J.-S. Guo, C.-T. Ling, and P. Souplet, "Non-self-similar deadcore rate for the fast diffusion equation with strong absorption," Nonlinearity, vol. 23, no. 3, pp. 657-673, 2010.

[7] J.-S. Guo and P. Souplet, "Fast rate of formation of dead-core for the heat equation with strong absorption and applications to fast blow-up," Mathematische Annalen, vol. 331, no. 3, pp. 651-667, 2005.

[8] J.-S. Guo and C.-C. Wu, "Finite time dead-core rate for the heat equation with a strong absorption," The Tohoku Mathematical Journal, vol. 60, no. 1, pp. 37-70, 2008.

[9] I. Stakgold, "Reaction-diffusion problems in chemical engineering," in Nonlinear Diffusion Problems, vol. 1224 of Lecture Notes in Mathematics, pp. 119-152, Springer, Berlin, Germany, 1986.

[10] J.-S. Guo, H. Matano, and C.-C. Wu, "An application of braid group theory to the finite time dead-core rate," Journal of Evolution Equations, vol. 10, no. 4, pp. 835-855, 2010.

[11] M. A. Herrero and J. J. L. Velázquez, "Explosion de solutions d'équations paraboliques semilinéaires supercritiques,"
Comptes Rendus de l'Académie des Sciences I. Mathématique, vol. 319, no. 2, pp. 141-145, 1994.

[12] N. Mizoguchi, "Blow-up rate of type II and the braid group theory," Transactions of the American Mathematical Society, vol. 363, no. 3, pp. 1419-1443, 2011.

[13] J.-S. Guo and B. Hu, "Blowup rate estimates for the heat equation with a nonlinear gradient source term," Discrete and Continuous Dynamical Systems A, vol. 20, no. 4, pp. 927-937, 2008.

[14] Z. C. Zhang, "Gradient blowup rate for a viscous HamiltonJacobi equation with degenerate diffusion," Archiv der Mathematik, vol. 100, no. 4, pp. 361-367, 2013.

[15] Z.-C. Zhang and B. Hu, "Boundary gradient blowup in a semilinear parabolic equation," Discrete and Continuous Dynamical Systems A, vol. 26, no. 2, pp. 767-779, 2010.

[16] Z. C. Zhang and B. Hu, "Rate estimates of gradient blowup for a heat equation with exponential nonlinearity," Nonlinear Analysis. Theory, Methods \& Applications, vol. 72, no. 12, pp. 4594-4601, 2010.

[17] Z. C. Zhang and Y. Li, "Blowup and existence of global solutions to nonlinear parabolic equations with degenerate diffusion," Electronic Journal of Differential Equations, vol. 2013, no. 264, 17 pages, 2013.

[18] Z. C. Zhang and Y. Y. Li, "Boundedness of global solutions for a heat equation with exponential gradient source," Abstract and Applied Analysis, vol. 2012, Article ID 398049, 10 pages, 2012.

[19] Z. C. Zhang and Y. Y. Li, "Gradient blowup solutions of a semilinear parabolic equation with exponential source," Communications on Pure and Applied Analysis, vol. 12, no. 1, pp. 269280, 2013.

[20] Z. C. Zhang and Z. J. Li, "A note on gradient blowup rate of the inhomogeneous Hamilton-Jacobi equations," Acta Mathematica Scientia, vol. 33, no. 3, pp. 678-686, 2013.

[21] L. P. Zhu and Z. C. Zhang, "Rate of approach to the steady state for a diffusion-convection equation on annular domains," Electronic Journal of Qualitative Theory of Differential Equations, vol. 39, no. 2, 10 pages, 2012.

[22] M. S. Floater, "Blow-up at the boundary for degenerate semilinear parabolic equations," Archive for Rational Mechanics and Analysis, vol. 114, no. 1, pp. 57-77, 1991.

[23] A. A. Lacey, "The form of blow-up for nonlinear parabolic equations," Proceedings of the Royal Society of Edinburgh A. Mathematics, vol. 98, no. 1-2, pp. 183-202, 1984.

[24] C. P. Wang and S. N. Zheng, "Critical Fujita exponents of degenerate and singular parabolic equations," Proceedings of the Royal Society of Edinburgh A. Mathematics, vol. 136, no. 2, pp. 415-430, 2006.

[25] C.-C. Wu and Z. C. Zhang, "Dead-core rates for the heat equation with a spatially dependent strong absorption," Discrete and Continuous Dynamical Systems B. A Journal Bridging Mathematics and Sciences, vol. 18, no. 8, pp. 2203-2210, 2013.

[26] J.-S. Guo, C.-S. Lin, and M. Shimojo, "Blow-up for a reactiondiffusion equation with variable coefficient," Applied Mathematics Letters, vol. 26, no. 1, pp. 150-153, 2013. 


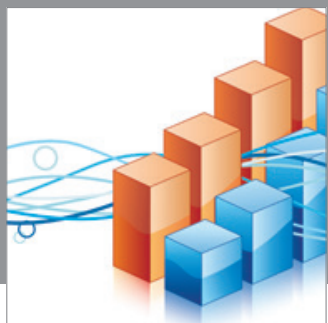

Advances in

Operations Research

mansans

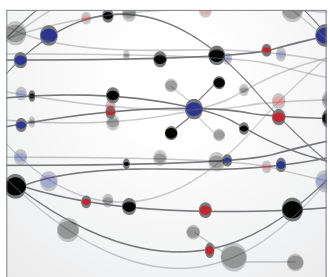

The Scientific World Journal
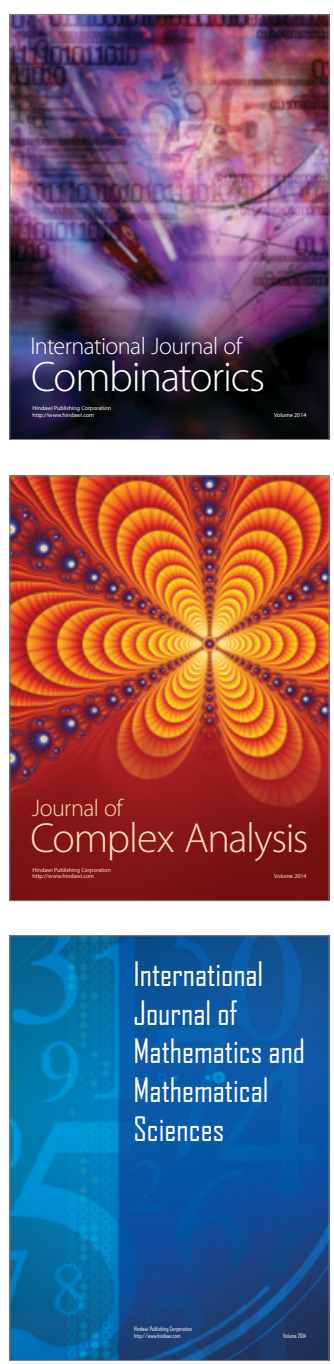
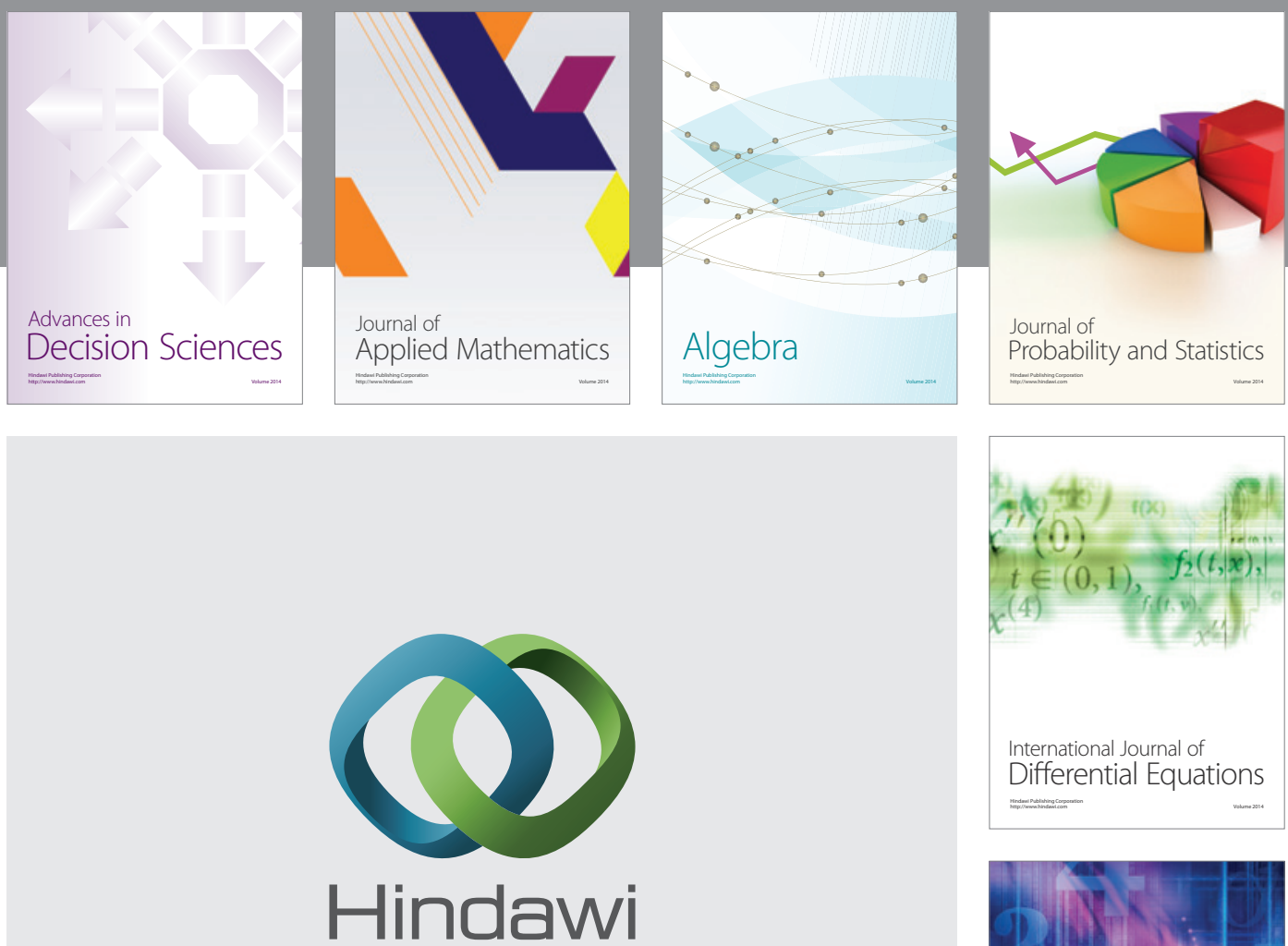

Submit your manuscripts at http://www.hindawi.com
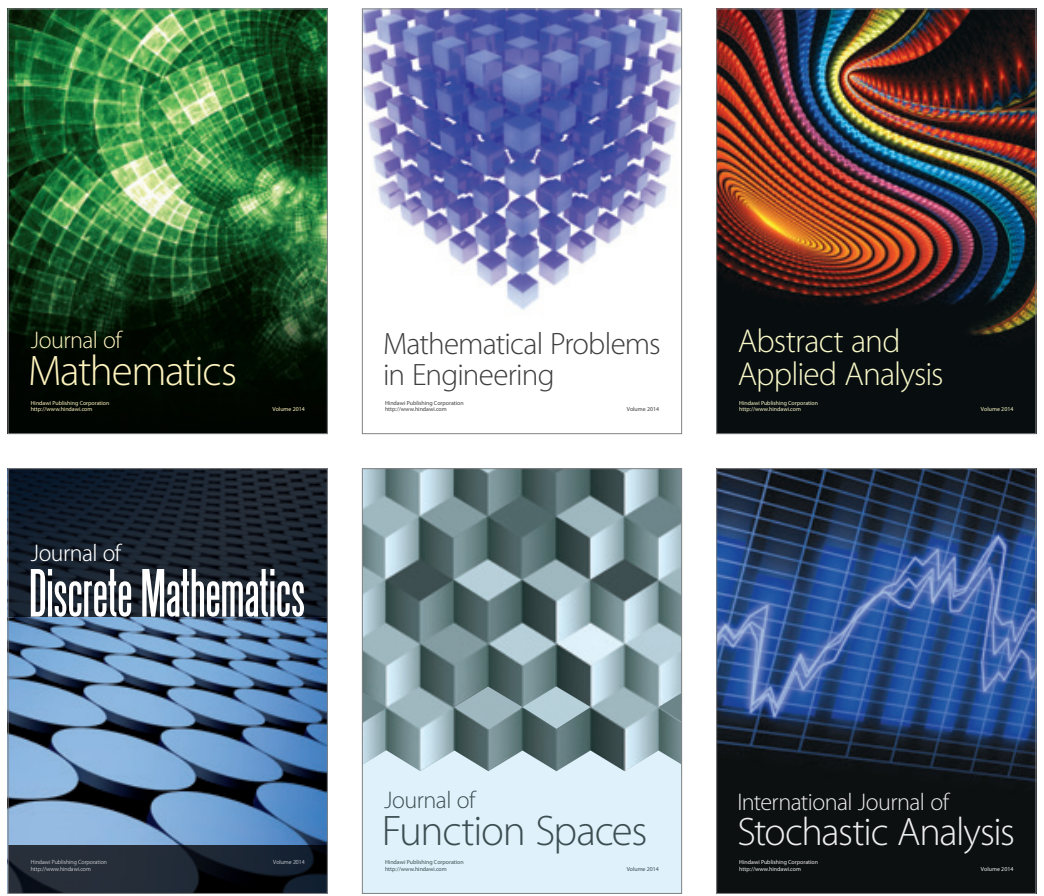

Journal of

Function Spaces

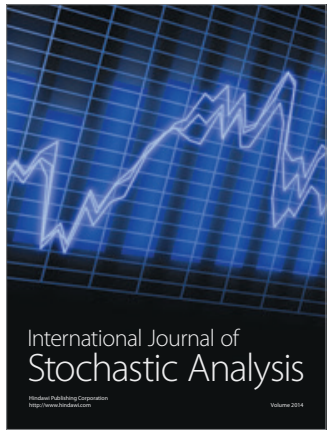

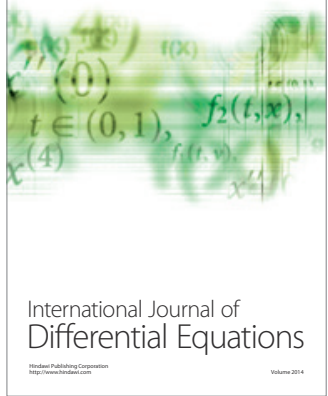
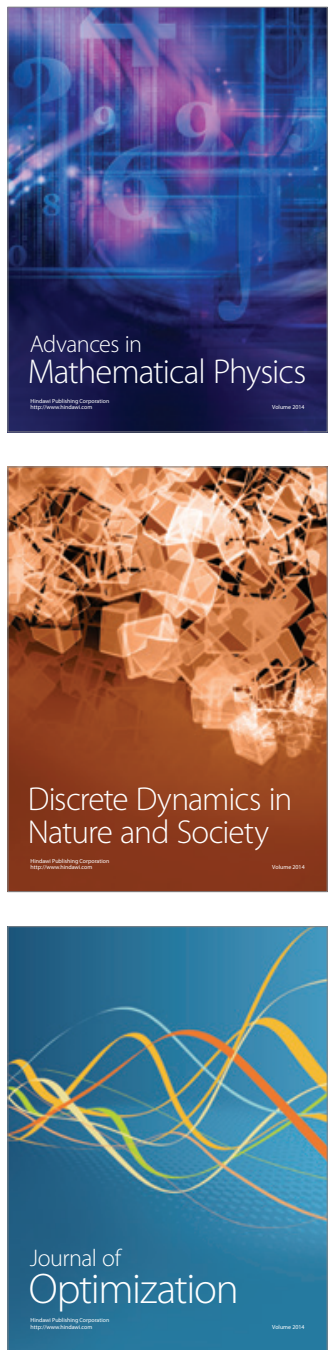